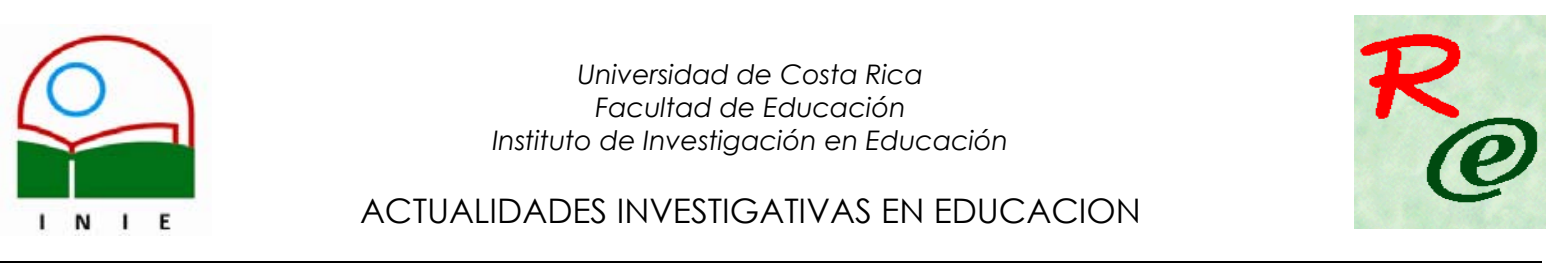

\title{
PROYECTO PROMOCIÓN DE LA CALIDAD Y LA EXCELENCIA: UNA EXPERIENCIA DESDE EL ASESORAMIENTO
}

\author{
Elsiana Guido Guido ${ }^{1}$ \\ Marianela Aguilar Arce ${ }^{2}$
}

Resumen: Durante el año 2003, el Centro de Evaluación Académica (CEA) lideró el Proyecto Promoción de la Calidad y la Excelencia, como un proceso de sensibilización, capacitación y reflexión sobre los aspectos relacionados con la autoevaluación de carreras, en relación con el mejoramiento y la acreditación. En este sentido, se buscaron espacios para discutir el significado de los procesos de autoevaluación; se realizaron actividades para informar, discutir y sensibilizar a la comunidad universitaria. Además, se generaron formas novedosas para capacitar a las personas, tanto del sector académico como administrativo, en la conducción de procesos de autoevaluación, autorregulación y en la preparación para la acreditación.

En este artículo, se presenta el desarrollo del Proyecto Calidad y Excelencia en la Universidad de Costa Rica, en lo que respecta a su planeamiento, ejecución y principales resultados. Se describe la participación que tuvieron, dentro del mismo, el personal profesional del CEA, los y las docentes de unidades académicas que ya habian desarrollado el proceso de autoevaluación y del personal docente de carreras acreditadas.

Según las autoras, mediante el desarrollo de este proyecto, fue posible poner sobre la mesa de discusión, la redefinición del concepto de asesoría académica que brinda el personal profesional del Departamento de Investigación y Evaluación Académica a las unidades académicas.

\section{Palabras clave: ASESORÍA ACADÉMICA/ AUTOEVALUACIÓN/ ACREDITACIÓN/}

Abstract: In 2003, the Academic Evaluation Center, AEC (Centro de Evaluación Académica, CEA), leads the Project promotion of quality and excellence, as a process of becoming aware, training and thinking about aspects related to the self-evaluation of careers, with a view to the improvement and with regard to the accreditation. In this sense, periods of discussion were search to analyze the meaning of the self-evaluation processes. Moreover, the AEC developed activities to inform, discuss and make aware the community of the University, as well as creative ways to train people from the academic and administrative sectors, in the management of self-evaluation processes, self-regulation and in the training for accreditation.

This article presents the development of the Project promotion of quality and excellence in the University of Costa Rica, with regard to its approach, implementation and main results. It describes the participation that the professional staff of the AEC, the professors from the Academic Units that had already developed the process of self-evaluation and of the teaching staff of accreditated careers had, within the said project.

According to the authors, through the development of this project it was possible to submit to discussion the redefinition of the notion of academic consultancy that the professionals of the Department of Research and Academic Evaluation offer to the Academic Units.

Key words: ACADEMIC CONSULTANT'S OFFICE/ AUTOEVALUACIÓN/ ACCREDITATION/

\footnotetext{
1 Politóloga y administradora de programas educativos no formales, de la Universidad de Costa Rica. Investigadora del Departamento de Investigación y Evaluación Académica del Centro de Evaluación Académica de la Universidad de Costa Rica. Investigadora en el Consejo Centroamericano de Acreditación de la Educación Superior. Correo electrónico: eguido@cariari.ucr.ac.cr

${ }^{2}$ Licenciada en Relaciones Internaciones de la Universidad Nacional de Costa Rica. Diplomado Superior en Ciencias Sociales con Énfasis en Relaciones Internacionales de la Facultad Latinoamericana de Ciencias Sociales FLACSO, Costa Rica. Exdirectora del Centro de Evaluación Académica, Universidad de Costa Rica. Actualmente se desempeña como Directora Ejecutiva del Consejo Centroamericano de Acreditación de la Educación Superior. Correo electrónico: maaguila@cariari.ucr.ac.cr
}

Artículo recibido: 14 de julio, 2005

Aprobado: 19 de setiembre, 2005 


\section{Presentación ${ }^{3}$}

Durante el año 2003, cuando fue diseñado y ejecutado el Proyecto Promoción de la Calidad y la Excelencia en la Universidad de Costa Rica (UCR), las instituciones de educación superior se encontraban inmersas en una coyuntura, nacional e internacional, que les planteaba el reto de responder a una diversidad de demandas por el mejoramiento de la calidad académica.

En la UCR se inició, desde mediados de los años noventas, una serie de experiencias en el ámbito de la autoevaluación con el fin de mejorar la calidad académica. En estas experiencias, participaron algunas unidades académicas y una Sede Universitaria en el proyecto denominado Cre-Columbus.

Dentro de este compromiso por el mejoramiento de la calidad, el papel de las asesorías brindadas por el Departamento de Investigación y Evaluación Académica (DIEA), del Centro de Evaluación Académica (CEA), a los procesos de autoevaluación, tuvo que redefinirse básicamente por dos motivos: por un lado, hacer más independientes y autosuficientes dichos procesos, desde la perspectiva de los propios gestores de la evaluación y, por otra parte, debido a las crecientes demandas de las Unidades Académicas (UA) ${ }^{4}$, que solicitaban un acompañamiento del personal del DIEA en los procesos de autoevaluación.

Es así cómo este artículo presenta dos propósitos fundamentales: en primera instancia, sistematizar y recuperar la experiencia desarrollada en este proyecto que constituyó una propuesta institucional en el ámbito del mejoramiento de la calidad académica; y en segunda instancia, se pretende aportar insumos a las discusiones del DIEA, sobre el tema de la asesoría y el papel de la persona asesora en estos procesos.

Como respuesta a los propósitos señalados, este artículo se organiza en tres partes: perspectiva teórica, los antecedentes del proyecto y el desarrollo y resultados del proyecto.

\footnotetext{
${ }^{3}$ En el planeamiento del Proyecto Promoción de la Calidad y la Excelencia, participaron, además de las autoras del artículo, la Dra. Alicia Vargas, jefa del DIEA y la Br. Mónica Quirós, investigadora del DIEA.

${ }^{4}$ Cuando se menciona a la Unidad Académica se refiere también a la Escuela o lo que en otras universidades se denomina Departamento.
} 
En el primer apartado, se presenta una perspectiva teórica acerca del concepto de asesoría. No solamente se analiza el papel del asesor, sino también el entorno organizacional en el que se desarrolla dicha asesoría, caracterizada por los procesos de toma de decisiones asociados a los procesos de autoevaluación.

En el segundo apartado, se recogen las experiencias de capacitación y formación del personal del DIEA que antecedieron a la realización del proyecto. En una tercera parte, se describen propiamente los tres módulos del proyecto Promoción de la calidad y la excelencia en la Universidad de Costa Rica: módulo de sensibilización, módulo de información y módulo de formación.

A manera de conclusión, se presentan algunos comentarios finales que recogen valoraciones acerca del desarrollo del proyecto.

\section{La función asesora}

El papel del asesor en las universidades ha ido teniendo un giro, como bien lo afirma Antonio Bolívar (1999) de la Universidad de Chile. Este cambio ha consistido en pasar de concebir a la persona asesora, como un agente de cambio externo, portadora de un enfoque más técnico o aplicativo, a una persona cuya función es facilitar cambios curriculares y organizativos.

Es en este sentido que Bolívar $(1999$, ๆ 5) nos habla de dos concepciones del papel asesor, por un lado se tiene

...la de un técnico especialista o experto que domina determinados contenidos o habilidades en función de las cuales puede/debe resolver los problemas que, según su especialización, se le planteen. Actúa como reacción a demandas por clientes individuales [...] desde un modelo de intervención único.

Y por otro lado, presenta un enfoque alternativo, según el cual, la persona asesora en lugar de presentarse como experto que -a la "demanda"- diagnostica los problemas [...] y propone soluciones [...], el asesor -como agente de cambio- trabaja conjuntamente con los profesores y profesoras, ya sea actuando como mediador entre el conocimiento pedagógico y los establecimientos, ya como colaborador en la 
identificación y solución de problemas, ejerciendo su principal función como dinamizador de los procesos de toma de decisiones del profesorado. (Bolívar, 1999, ๆ 6)

Dentro de este segundo enfoque de asesoramiento, se supera la visión tradicional del estilo clínico de asesoramiento, esto es, la de una persona experta que aporta soluciones a los problemas, como si se tratara de una receta médica. Lo que se propone, más bien, es el establecimiento de una relación de colaboración en el trabajo entre las personas asesoras y las que solicitan sus servicios profesionales. Según Bolívar $(1999$, I 9$)$ esta colaboración "supone crear una relación de igualdad entre asesores/as y profesores/as para que el intercambio comunicativo provoque una reflexión conjunta de la situación, deliberando de manera compartida sobre la situación y sobre las decisiones a tomar".

Hernández (1992) aporta insumos importantes que ayudan a perfilar el papel del asesor, dentro del contexto de la educación. El autor señala que el o la asesora cuenta con una capacidad reflexiva que ayuda al análisis de la práctica profesional y de las relaciones que se establecen en las UA, de manera tal, que la construcción de la realidad se establece, mediante este intercambio entre el personal asesor y las personas que son asesoradas. Esta conceptualización permite superar visiones, en las cuales, la persona asesora es quien dice qué debe hacerse y establece, de manera lineal, los procedimientos y acciones por seguir.

R. Bolam citado por Hernández (1992) define la función del asesor, como la del profesional que "trabaja junto con los departamentos de educación y las escuelas para ayudarles a establecer sus propios procedimientos para plantear y resolver problemas y tomar decisiones en torno a ellos"

Jesús D. Segovia (2003) argumenta a favor de este nuevo enfoque alternativo en el asesoramiento universitario, al proponer que el tipo de asesoramiento que implica el establecimiento de relaciones verticales con las personas asesoradas, viene fracasando en la educación. Con este tipo de asesoramiento externo, la persona que asesora ejerce una función supervisora y se convierte "en el primer instrumento político claramente invasivo, en relación con la práctica profesional". Este autor de la Universidad de Granada, en España, 
presenta el asesoramiento como una cuestión de poder, de saber y de identidad y, a este tipo de asesoramiento, lo denomina "poder del experto".

Frente a este tipo de poder del experto, Segovia (2003) propone que se establezca un cierto equilibrio entre las relaciones de poder que siempre se establecen en los intercambios entre los profesionales. De esta manera, se debe "dosificar con equidad el ejercicio del saber como poder, con el de la autonomía y profesionalidad del centro y sus profesores, hasta llegar a establecer un sabio y productivo consenso". Con este planteamiento, se superaría la visión de la persona asesora como la dueña del saber, portadora de soluciones y buscadora de problemas.

Si bien es cierto, no debe existir una relación vertical en el asesoramiento, tampoco debe caerse en el otro extremo de subvalorar el conocimiento y la experiencia de las personas técnicas, ya que estas pueden aportar, mediante las capacitaciones, una visión general de los procesos la cual conlleve a un trabajo más independiente de las UA. Es en este sentido que Antonio Bolívar (1999, ๆ 15) considera que la capacitación constituye una función muy importante, dentro de la labor asesora. El autor afirma que debe favorecerse la planificación de las acciones formativas, ya que mediante éstas, es posible aprender de la práctica. "En lugar de proporcionar medios o recursos puntuales, a la larga es más exitoso capacitar al profesorado para que pueda resolver, por sí mismo, los problemas cotidianos que, en estos ámbitos, se vayan presentando".

\subsection{Cultura organizacional, asesoramiento y toma de decisiones}

Para que la persona asesora colabore con la reflexión en las UA, es importante el conocimiento que tenga, acerca del marco organizacional en el que se desenvuelven las personas que requieren del apoyo para construir sus proyectos en el ámbito académico.

Es sabido que toda institución u organización tiene un marco normativo que orienta el desarrollo de los procesos que lleva a cabo. Sin embargo, no sólo el marco normativo constituye la guía para la toma de decisiones, sino también las características particulares de la organización, surgidas de las prácticas cotidianas y de las interacciones entre las personas. 
Para la persona que realiza la función de asesoramiento en una organización y, en este caso, específico en UA de una institución universitaria, es indispensable conocer las características particulares que distinguen los procesos de toma de decisiones en cada Escuela, es decir, conocer la cultura de ese lugar, el contexto en donde, mediante ciertos códigos, se llevan a cabo los procesos académicos. Sobre este aspecto, el profesor Mathias Wesseler, de la Universidad de Kassel, en Alemania, señala:

Universidades, facultades y departamentos, hasta las disciplinas científicas mismas tienen sus propias culturas. $Y$ son las culturas las que - muchas veces inconscientemente - deciden sobre las acciones. Las culturas, con sus diferencias, inciden clandestinamente en los detalles de la labor diaria universitaria... (2004, p.86).

Este marco de lo formal, es decir, de lo normativo y de lo informal, visto desde los procedimientos y costumbres, es lo que se conoce como cultura organizacional. A partir de la definición aportada por varios autores, el profesor Wesseler construye su propia definición de la cultura organizacional, la cual se entiende como "....un sistema de expectativas de lo que es una conducta o creencia apropiada de los miembros de una institución. La cultura forma una matriz que delinea roles, aspiraciones, actitudes, normas y valores“ (2004, p. 86).

Además de conocer parte de la cultura organizacional, la persona que asesora debe conocer la manera cómo se toman las decisiones en las instancias de educación superior. En instituciones como la Universidad de Costa Rica, los procesos de toma de decisiones son complejos, pese a que la institución tiene claramente plasmados, en el Estatuto Orgánico, los ejes sustantivos del modelo universitario, a saber: la docencia, la investigación y la acción social. No podría afirmarse que por poseer esos lineamientos, exista una tendencia a la homogenización en cada una de las UA y unidades administrativas de la Universidad. Por el contrario, al parecer la misma estructura y organización universitaria tiende a consolidar las particularidades y las diferencias, ya que la institución universitaria es vista como "un sistema débilmente acoplado" que se organiza políticamente, en donde "...cada unidad académica tiene su propia dinámica y su relativa independencia, ya sea para incorporar cambios e innovaciones o para permanecer rezagada, con repercusiones poco significativas para el resto de la institución" (Vargas, 2001, p. 306). 
En cada UA existen normas y reglas aprendidas, o subculturas, que como bien lo anota el profesor Wesseler, se evidencian no sólo entre las disciplinas científicas, sino también entre los ámbitos académicos, vinculados con la docencia, la investigación y la extensión, con lo administrativo (2004, p. 88). Las interacciones que se desprenden de estas subculturas oscilan entre la tensión, la lucha, la indiferencia, la coexistencia y la cooperación.

De lo anterior, se desprende que la persona asesora debe tener siempre una actitud receptiva y flexible, que le permita conocer, en primera instancia, el contexto de la UA, así como las características del desarrollo de la disciplina. En las primeras sesiones, es importante indagar ¿qué le interesa a esta Escuela?, ¿cuál es el estilo con el que realizan sus procedimientos y cómo se toman las decisiones?

El asesor o la asesora debe aprovechar las prácticas y conocimientos de las personas que integran las UA para enlazarlos con sus propios conocimientos. De este modo, la función asesora implica, como bien lo apunta Bolívar (1999), conocer las propuestas, contenidos y procesos que puedan contribuir a la resolución de problemas.

En los proyectos de autoevaluación, la asesoría que se brinda está orientada a propiciar la reflexión que conduzca a una toma de decisiones mejor informada. Dentro de esto, el asesor o la asesora ejerce una función de facilitador de los procesos y facilitador de la negociación y la construcción de acuerdos entre las partes, al propiciar las discusiones y agregar insumos importantes para la reflexión.

La acción facilitadora y la negociación son promovidas por la persona asesora, partiendo de una concepción de asesoría no lineal ni vertical, sino más bien, mediante un estilo de asesoría de tipo horizontal, y no clínico, en los términos en que se explicó anteriormente.

La función de negociación se impulsa por medio de dos acciones fundamentales a saber, proporcionar insumos o información y facilitar la comunicación y discusión entre los integrantes de la comunidad académica; con el propósito de plantear acuerdos concretos que produzcan avances específicos en los procesos que se lleven a cabo, así como a la toma de decisiones pertinentes. 
Dentro del modelo de asesoramiento que promueve las relaciones horizontales entre los diferentes profesionales, en la siguiente figura, se esquematizan los vínculos que se establecen entre la persona que asesora y las UA de la Universidad.

Figura No. 1

Papel de la persona asesora en el proceso dinamizador de toma de decisiones

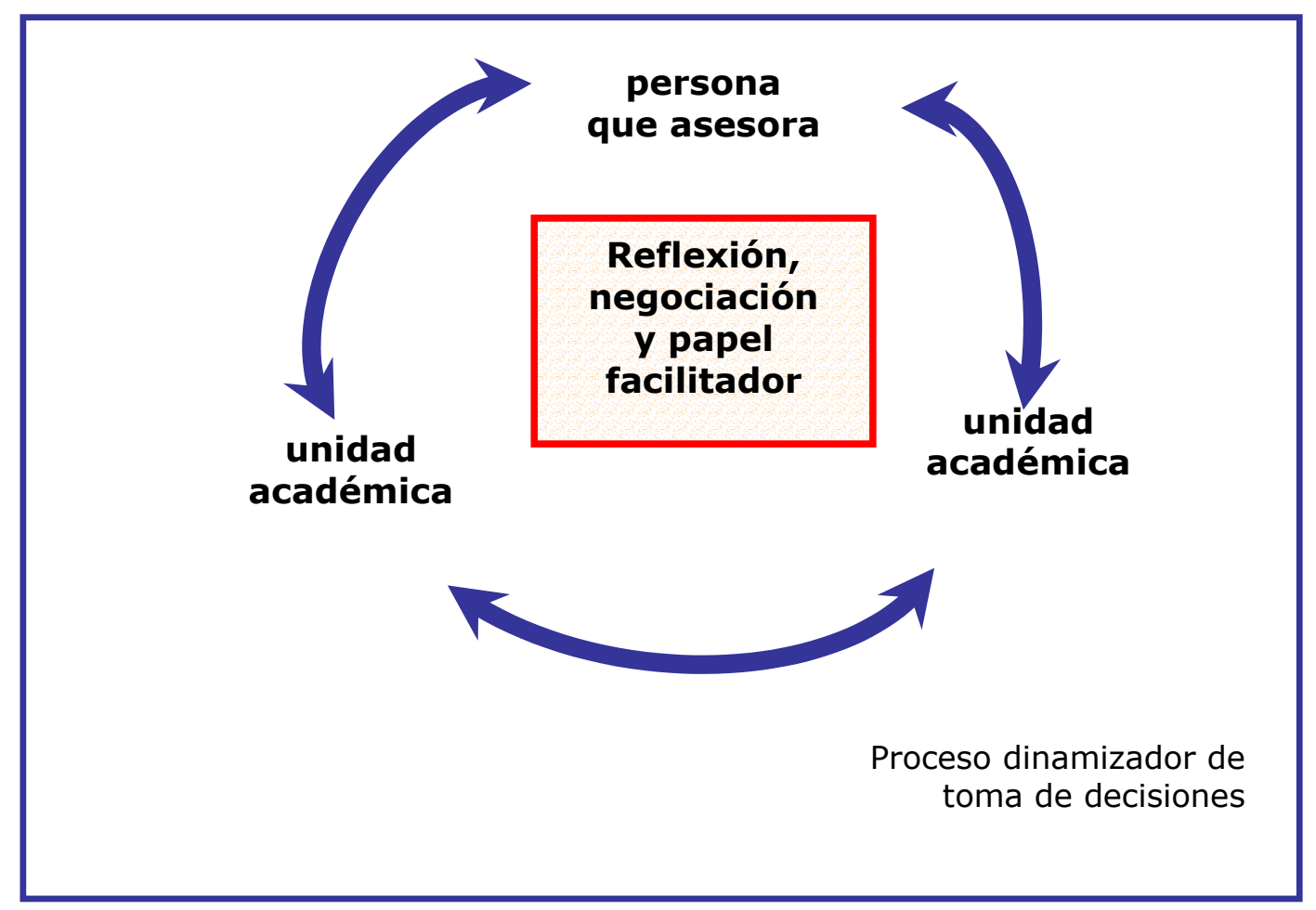

Esquema elaborado por las autoras del artículo.

En la figura anterior se destaca el papel facilitador de la persona asesora, en la reflexión y la negociación para la toma de decisiones. Es importante hacer notar que no solo se ejerce este papel facilitador con las UA que acompaña en el proceso, sino que también propicia espacios para que se dé una relación de aprendizaje entre UA que han tenido experiencias en los procesos de autoevaluación y que también contribuyen a la función de asesoramiento, por medio de la socialización de sus experiencias con otras UA. Estas relaciones de cooperación entre ellas, evidencian que la persona asesora se posiciona, no como la poseedora del conocimiento y la única que puede aportar insumos para la solución de los problemas. 


\section{Algunos antecedentes del Proyecto Promoción de la Calidad y la Excelencia en la Universidad de Costa Rica}

\subsection{Proceso de formación y capacitación del personal del Departamento de Investigación y Evaluación Académica (DIEA)}

EI DIEA asumió la función de acompañar los procesos de autoevaluación en la Universidad de Costa Rica. Estos procesos, precisamente por su carácter novedoso, implican un abordaje constructivo y un aprendizaje conjunto entre las UA participantes y el personal del Departamento (DIEA).

Ante la creciente demanda de apoyo de las UA a sus procesos de autoevaluación, la Dirección del CEA y la Jefatura del DIEA planearon y organizaron un programa sistemático de capacitaciones. La intención era fortalecer internamente al DIEA, al construir y consensuar ejes y fundamentos básicos para la asesoría evaluativa. Adicionalmente se buscaba mejorar la calidad de los asesoramientos y del acompañamiento que se brindaba a las Escuelas.

El personal del DIEA participó en diversas capacitaciones y cursos, nacionales e internacionales, con los que se pretendía construir una base conceptual que incluyera herramientas teóricas, técnicas y metodológicas, con las cuales hacerle frente a las funciones de asesoría en temas relativos al mejoramiento de la calidad del quehacer académico universitario.

Los esfuerzos por lograr una mejor formación del personal del DIEA, se pueden agrupar en dos grandes ámbitos: uno que se desarrolló durante los años 2002 y 2003 en el Centro de Evaluación Académica, en donde se programaron sesiones de estudio, análisis y talleres para el replanteamiento de la Guía de autoevaluación con que contaba el CEA. El otro, proviene de una iniciativa centroamericana coordinada por el Consejo Superior Universitario Centroamericano (CSUCA) y apoyado por la cooperación alemana.

Gran parte de las capacitaciones, realizadas en el marco del CSUCA y de la cooperación alemana, abarcaron diversas áreas, a saber: métodos para la autoevaluación, papel de los 
pares evaluadores y la gestión universitaria para enfrentar los retos actuales, entre otras. Seguidamente se describen los alcances de algunas de estas iniciativas:

a. En el Curso CAMINA-COSTA RICA 2002, se capacitaron siete personas del DIEA. Este curso interuniversitario, denominado Curso de capacitación en métodos innovativos de autoevaluación, estuvo dirigido al personal técnico de las unidades de evaluación y a algunos miembros de las comisiones de autoevaluación de las cuatro universidades estatales. También se integraron personas de las cuatro universidades privadas que venían participando en el Sistema Nacional de Acreditación de la Educación Superior (SINAES).

Uno de los requisitos de aprobación del curso fue el desarrollo de investigaciones en temas relativos a la autoevaluación en las universidades participantes. De esta manera, durante un año fueron se desarrollaron investigaciones cuyos objetivos principales consistían en mejorar los conocimientos y metodologías para las asesorías que se brindan a las UA en los procesos de evaluación. Se debe mencionar que anteriormente, en el año 2000, dos personas del DIEA habían participado en el primer curso de capacitación de CAMINA 2000, ofrecido por el CSUCA y la cooperación alemana al personal académico de la región centroamericana. Estas personas fueron parte de los organizadores y facilitadores de la versión CAMINA-Costa Rica.

b. En esta misma línea de formación, otra funcionaria del DIEA participó en el curso UNISTAFF (University Staff Development Programme). Este curso fue diseñado para enfrentar los más urgentes retos de las universidades en el ámbito mundial. El programa, en el que participó el personal académico y administrativo de instituciones de educación superior de África, Asia y Latinoamérica, ofreció un espacio para compartir experiencias desarrolladas en diversos países, discutir aspectos teóricos de la educación superior y analizar los diversos problemas del desarrollo organizacional de las universidades. El programa se realizó con el apoyo del DAAD (Servicio Alemán de Intercambio Académico), la Fundación Alemana para el Desarrollo Internacional (DSE), el Instituto de Estudios Socioculturales (ISOS) de la Universidad de Kassel en Alemania y contó con la colaboración de la Universidad de Costa Rica.

c. Con el fin de apoyar la gestión de los procesos de autoevaluación, se capacitó como evaluadores externos, a un grupo conformado por personal del DIEA y por docentes de las 
UA que estaban en el proceso de recibir la visita de los pares evaluadores. Dos grupos importantes de funcionarios del DIEA, tuvieron la oportunidad de formarse como pares evaluadores; unos en cursos internacionales y, el grupo más numeroso, mediante la capacitación que brindaron los pares evaluadores de las cuatro universidades estatales, formados por el CSUCA.

d. En el área de sostenibilidad de los procesos de autoevaluación, profesores alemanes ofrecieron una capacitación a universidades estatales y privadas costarricenses. Esta capacitación en metodologías innovadoras, estaba dirigida a promover, en académicos y académicas, los estudios de seguimiento de graduados, como un instrumento de gestión de calidad. La capacitación no sólo tenía como objetivo, compartir las metodologías para dichos estudios, sino también trabajar en la sistematización de la información, mediante la organización y elaboración de bases de datos de las personas graduadas.

Gran parte de estas opciones de capacitación, fue posible, debido a los convenios de cooperación que el Gobierno alemán brinda a la región centroamericana, por medio del CSUCA. Iris Erazo, coordinadora de la Comisión Técnica del Sistema Centroamericano de Evaluación y Acreditación de la Educación Superior (SICEVAES) presenta como un logro importante de la metaevaluación del SICEVAES, precisamente lo alcanzado en los

...procesos de capacitación y entrenamiento de personal, diseño y desarrollo de guías y materiales de evaluación, implementación de procesos de auto-evaluación y evaluación externa y, al final pero no por ello menos importante, el desarrollo de instancias de reflexión, evaluación y análisis de las experiencias realizadas. (...) así como el desarrollo de metodologías de capacitación y entrenamiento innovadoras y eficaces, que pueden servir de modelo a otros procesos tanto en la región como fuera de ella. Asimismo, el énfasis en el desarrollo de la región como un todo, unido al apoyo concreto prestado a cada uno de los países constituye también un elemento innovador y sugerente para otras regiones con características similares. $(2005, \mathrm{p} .12)$

De este modo, las diversas capacitaciones recibidas propiciaron que el personal del DIEA, pudiera entrar en un productivo proceso de reflexión y de aprendizaje, en torno a los temas de evaluación y calidad académica. Adicionalmente, los resultados de las investigaciones realizadas, así como del estudio individual y colectivo sobre distintos documentos y temas 
relacionados, constituyeron el marco idóneo para el desarrollo del Proyecto promoción de la calidad y la excelencia en la Universidad de Costa Rica.

\section{Desarrollo y principales resultados del Proyecto Promoción de la Calidad y la Excelencia en la Universidad de Costa Rica}

A mediados de los noventa, el CEA fue la instancia que debió asumir parte de los retos que, en materia de la promoción de la calidad académica, se encontraban plasmados en las políticas emitidas por el Consejo Universitario, concretamente las propuestas de ejecución contempladas en el Plan de trabajo de la Rectoría 2000-2004 y en la Resolución VD-R7042-2001 emitida por la Vicerrectoría de Docencia.

Junto a otras oficinas universitarias, el CEA se encargó de promover y liderar las discusiones que, en materia de autoevaluación, se estaban desarrollando en la Universidad de Costa Rica. Esto fue posible dadas la maduración teórica del pensamiento y la reflexión del personal del DIEA, lo que favoreció una toma de posición ante los temas de la autoevaluación y la acreditación de carreras.

Fue así cómo en el año 2003 se inició el proyecto titulado Promoción de la Calidad y la Excelencia en la Universidad de Costa Rica. Este proyecto contó con el apoyo de la Vicerrectoría de Docencia y fue inscrito, en esa instancia, como un proyecto de mejoramiento de la calidad académica.

Para el diseño del proyecto, la Dirección del CEA con la jefatura y el equipo del DIEA, se plantearon dos grandes propósitos: por una parte, articular institucionalmente distintos esfuerzos que, en materia de mejoramiento de la calidad académica, se estaban gestando, de manera dispersa, en el ámbito universitario. Por otra parte, se quería favorecer un desarrollo más autónomo de las UA en la conducción de sus procesos de autoevaluación, para potenciar, de este modo, el papel facilitador del personal asesor del DIEA.

Con tales intenciones, se formularon cuatro grandes objetivos en este proyecto:

1. Ofrecer un espacio a la comunidad universitaria para la reflexión, análisis y discusión del significado de los procesos de autoevaluación y autorregulación y de la acreditación en la UCR, en el marco de las necesidades institucionales y de las tendencias externas. 
2. Desarrollar una campaña divulgativa, utilizando diversos medios de comunicación, para informar y sensibilizar a la comunidad universitaria, sobre la importancia de los procesos de calidad que deberían asumir, voluntariamente, cada UA y cada miembro de la comunidad universitaria.

3. Abrir espacios que permitan la formación de personas de las distintas áreas académicas de la UCR, sobre cómo conducir los procesos de autoevaluación, autorregulación y cómo trabajar hacia la acreditación.

4. Involucrar a las UA que han llevado a cabo los procesos de autoevaluaciónautorregulación y la acreditación, para que sean garantes de los beneficios obtenidos con sus experiencias en los procesos de mejoramiento.

El proyecto se organizó en tres módulos: el módulo de sensibilización, de información y el de formación.

Figura No. 2

PROYECTO PROMOCIÓN DE LA CALIDAD Y LA EXCELENCIA

EN LA UNIVERSIDAD DE COSTA RICA

Módulos de sensibilización, información y formación

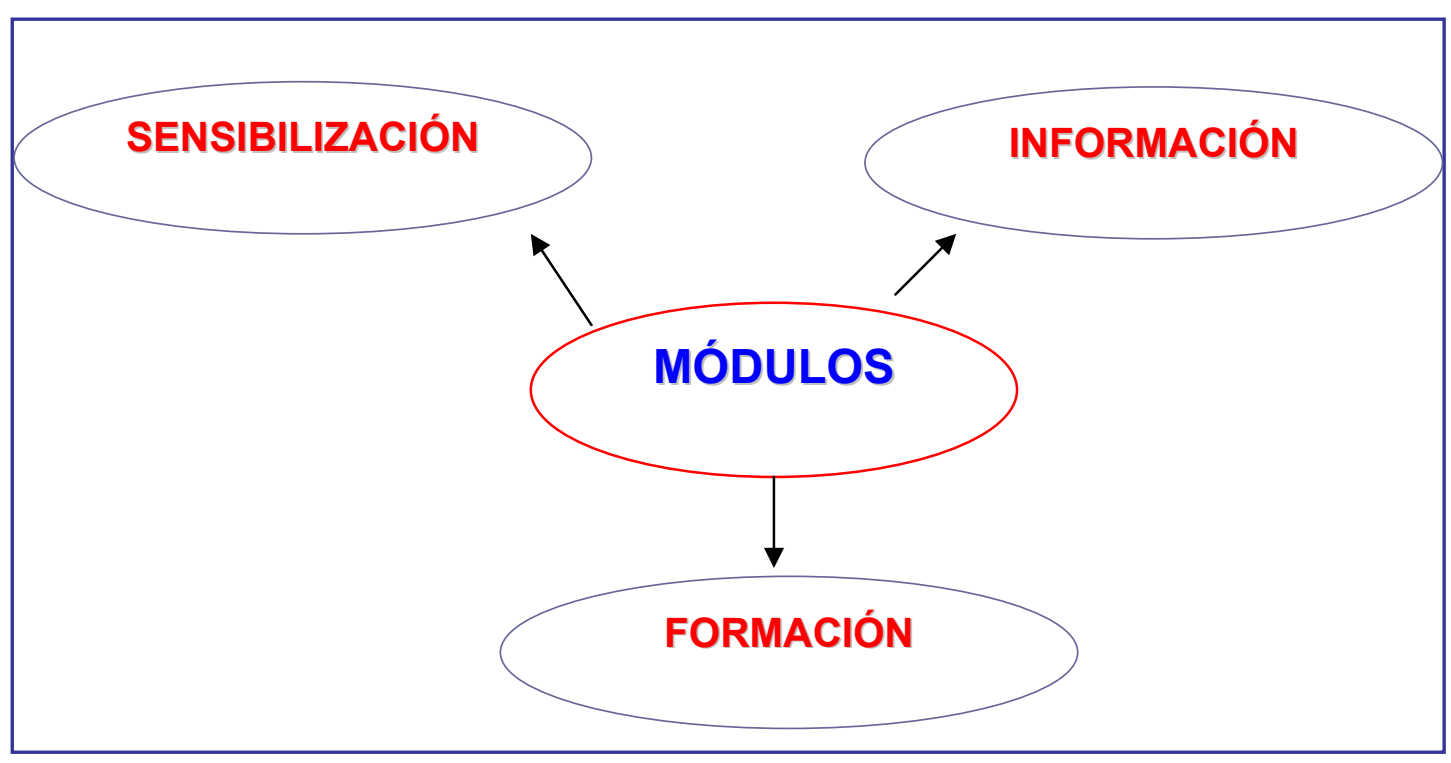

Elaboración propia. 


\subsection{Módulo de sensibilización}

Con este módulo, se pretendía buscar un acercamiento del CEA a las UA, por medio de los consejos asesores de facultad ampliados. El objetivo era conversar acerca de la importancia de los procesos de autoevaluación-autorregulación, la experiencia acumulada en la Universidad de Costa Rica, así como del apoyo del DIEA a tales procesos. Los consejos asesores de facultad están conformados por los directores y directoras de las UA y de los centros e institutos de investigación, también se cuenta con representación estudiantil y del profesorado, además del decano o decana, quien preside.

La siguiente figura muestra la organización del módulo de sensibilización, cuya estrategia de desarrollo fue mediante conversatorios:

Figura No. 3

\section{PROYECTO PROMOCIÒN DE LA CALIDAD Y LA EXCELENCIA}

EN LA UNIVERSIDAD DE COSTA RICA

Módulo de sensibilización

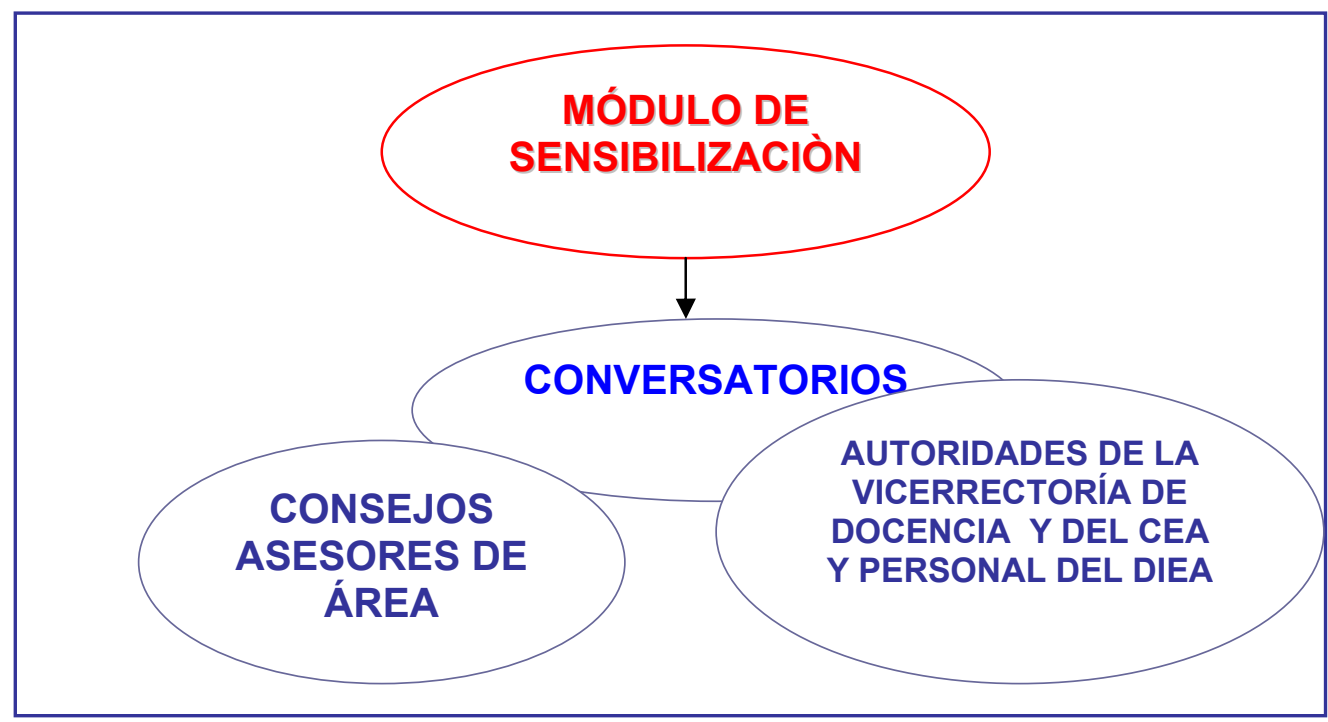

Elaboración propia.

\subsubsection{Conversatorios}

En el módulo de sensibilización, participaron autoridades de las decanaturas, direcciones de Escuela y del CEA, docentes-investigadores del DIEA y profesores y profesoras integrantes de los consejos asesores de facultad ampliados, de cada una de las facultades 
de la UCR. En el año 2003, se realizaron cuatro conversatorios con las Áreas de Ciencias Básicas, Ciencias Sociales, Ciencias Agroalimentarias y Ciencias de la Salud e Ingeniería.

Esta estrategia de conversatorios permitió un intercambio amplio y libre de información, no sólo acerca del apoyo que el CEA podía brindar al desarrollo de los procesos de autoevaluación, sino también en torno a las expectativas, intereses, malestares, críticas y recomendaciones que realizaban las autoridades de las distintas UA reunidas en esa instancia. Posterior a una breve exposición sobre el quehacer general del CEA, así como de los alcances del proyecto de calidad y excelencia, se abría un espacio para que los participantes externaran sus inquietudes y comentarios.

El logró fundamental de estos conversatorios, fue reflexionar y discutir con las autoridades universitarias, acerca del proceso de autoevaluación y su importancia para el mejoramiento de la calidad académica. Además se contribuyó a concienciar, acerca de la importancia de apoyar la gestión de estos procesos en las respectivas Escuelas.

Esos diálogos abiertos permitieron al DIEA, retroalimentar su trabajo, mediante el replanteamiento de algunos aspectos del quehacer de las asesorías que se brindan, y a las autoridades del CEA asegurarse de que el planeamiento anual, estaba adecuadamente orientado, de acuerdo con las necesidades e intereses de las UA. Este módulo de sensibilización propició un intercambio comunicativo y reflexivo, entre el personal docente y el asesor, lo cual permitió, como bien apunta Bolívar (1999), obtener insumos importantes para alimentar la toma de decisiones en el CEA.

\subsection{Módulo de información}

Este módulo tenía como objetivo informar y divulgar a la comunidad universitaria, los principales avances, tendencias y hallazgos, en materia de evaluación y calidad en la educación superior. Constituía un espacio abierto a toda la comunidad universitaria para la realización de conferencias dictadas por expertos en diferentes temas relacionados con la autoevaluación y la autorregulación. Dentro de este espacio, también se contemplaba un rol activo por parte de los y las profesionales del DIEA, mediante la presentación de resultados de las investigaciones realizadas en esos temas. Adicionalmente, se abrió un espacio para compartir la experiencia vivida por las UA que habían realizado procesos evaluativos, tendientes a la acreditación. 
Se presentaron cuatro modalidades diferentes de actividades, mediante, las cuales, se invitaba a la comunidad académica a discutir sobre estas temáticas de actualidad:

1. Conferencias y otras actividades informativas y de proyección impartidas por personas expertas en temas relacionados con la calidad académica.

2. Foro de discusión sobre las experiencias llevadas a cabo por las UA que habían realizado procesos de autoevaluación. En este foro, participaron las personas integrantes de las comisiones de autoevaluación de Escuelas que ya habían realizado estos procesos.

3. Presentación de resultados de investigaciones realizadas en el marco del proyecto CAMINA/COSTA RICA 2002, así como de otras llevadas a cabo en el DIEA, cuyas temáticas favorecían los procesos de autoevaluación y autorregulación de las UA.

4. Microprogramas informativos pautados en Radio Universidad.

La siguiente figura muestra la organización del módulo de información:

Figura No. 4

PROYECTO PROMOCIÓN DE LA CALIDAD Y LA EXCELENCIA EN LA UNIVERSIDAD DE COSTA RICA Módulo de información

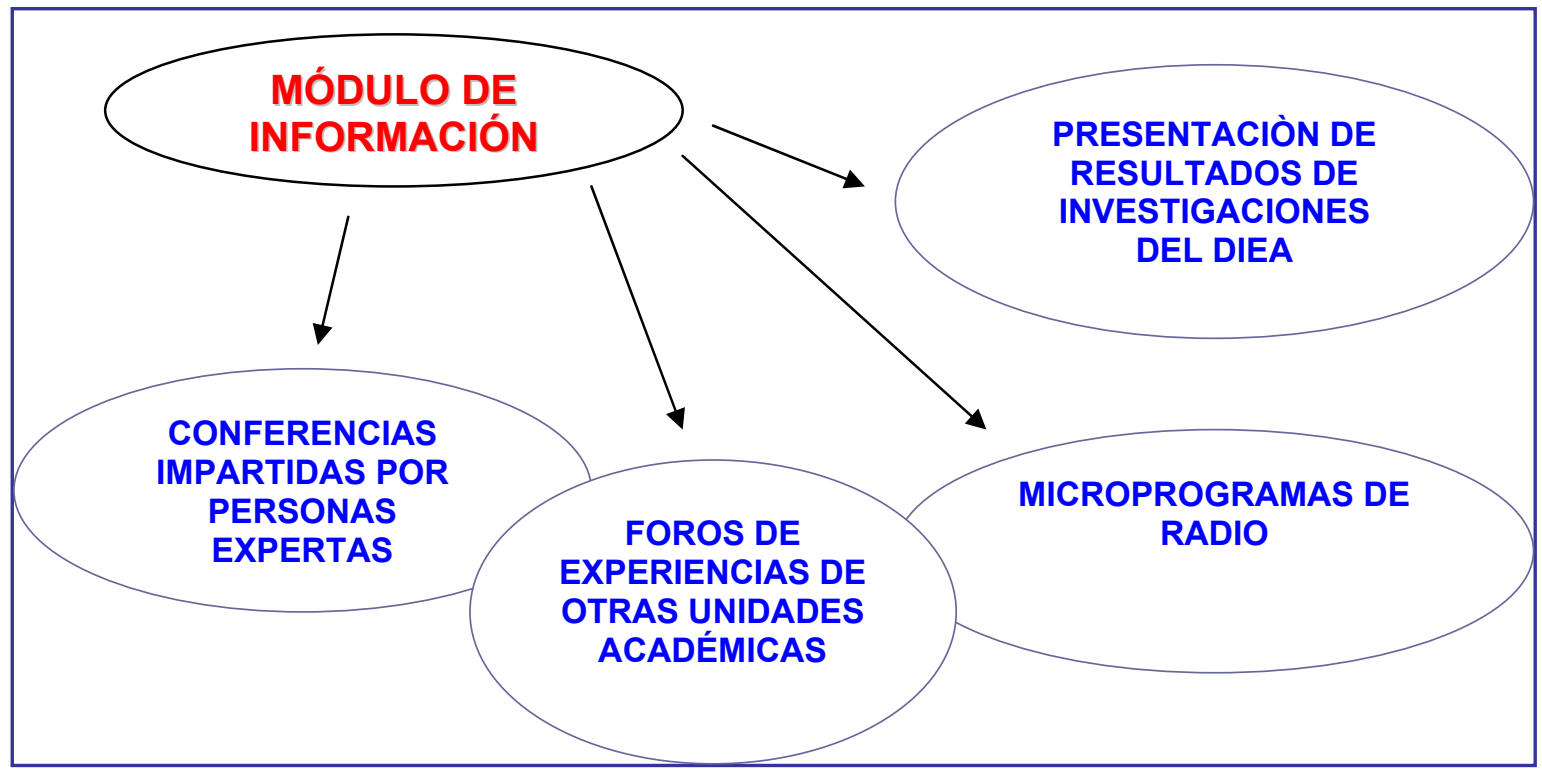

Elaboración propia. 


\subsubsection{Conferencias impartidas por personas expertas}

Se cursó invitación al Dr. Miguel Ángel Ripoll, académico chileno de la Universidad de la Frontera, especialista en Psicología y Evaluación Educativa, quien cuenta con una larga trayectoria en materia de evaluación universitaria. Con este especialista, se organizaron varias actividades: una conferencia magistral dirigida a la comunidad universitaria, denominada Cómo abordar los problemas metodológicos en los procesos de autoevaluación de carreras. A esta actividad, asistieron funcionarios de las unidades técnicas de evaluación de las universidades estatales y privadas. Además con el Dr. Ripoll, se organizó un taller interuniversitario sobre tratamiento de datos cuantitativos y cualitativos, dirigido a los equipos técnicos asesores de las cuatro universidades estatales. Este taller tenía como objetivo principal, brindar una capacitación sobre metodologías para la recolección y tratamiento de la información de los procesos de evaluación.

Específicamente para trabajar de manera conjunta con el personal del DIEA, se desarrollaron actividades adicionales que permitieron atender aspectos de interés para el CEA, particularmente sobre la rigurosidad y precisión de los instrumentos y sobre los procedimientos utilizados para la recopilación de la información en los procesos de autoevaluación. Es así cómo se llevó a cabo un taller sobre metodologías de evaluación y un conversatorio sobre instrumentos de autoevaluación, debido a que mediante la construcción de los instrumentos y el seguimiento de los procedimientos, se contribuye a garantizar la validez y confiabilidad del proceso de autoevaluación.

\subsubsection{Foros acerca de experiencias de Unidades Académicas}

EI CEA desarrolló foros acerca de las experiencias de las UA, de manera tal que, el conjunto de temas por tratar brindaran una perspectiva integral de las distintas facetas que engloban el proceso de autoevaluación. Es decir, desde la perspectiva de su planeamiento, organización de la información, hasta la consideración del aporte de los evaluadores externos, así como del impacto que, en el desarrollo de las UA, han tenido los procesos de autoevaluación, mejoramiento y la misma acreditación.

El planeamiento de esta actividad, se fundamentó en los resultados positivos obtenidos de otras actividades que, anteriormente, había organizado el CEA, en donde las UA adquirían un protagonismo relevante, mediante la interacción con otras Escuelas. En estos intercambios las UA contaban sus propias experiencias y aprendizajes a otras Escuelas, se 
propiciaba un mayor acercamiento así como un aprendizaje interactivo, al compartir procedimientos, metodologías, actitudes, planeamientos y experiencias de gestión, entre otros.

Para el CEA, esta modalidad de asesoría académica significó no solo una importante innovación, sino además un recurso de enseñanza y aprendizaje que favoreció la visualización de la asesoría, mediante el desarrollo de procesos más horizontales (EscuelaEscuela). Este aprendizaje además de ser provechoso para las comisiones de autoevaluación de las Escuelas que apenas iniciaban el proceso, lo fue también para el personal asesor del DIEA.

El Foro: experiencias de autoevaluación en la Universidad de Costa Rica, organizado por el CEA, constituyó la actividad mayor de este módulo. En él participaron representantes de las comisiones de autoevaluación de varias Escuelas y personal del DIEA. En esa oportunidad, el tema tratado por el representante del DIEA, fue el de la organización de la comisión para llevar a cabo las distintas etapas de la autoevaluación. La Escuela de Biología se encargó del tema ¿cómo se recopila y analiza la información en un proceso de autoevaluación? La Facultad de Farmacia desarrolló la temática referente a los aportes que brindan los pares académicos al proceso de evaluación y al plan de mejoramiento, y también se contó con la participación de un representante de la Escuela de Medicina, quien se encargó del tema acerca de los beneficios que ha traído la acreditación.

\subsubsection{Microprogramas de radio}

La tercera estrategia de este módulo fue constituida por microprogramas de radio, pautados en el espacio Con calidad y excelencia de Radio Universidad de Costa Rica, durante los meses de setiembre a diciembre del año 2003.

El objetivo de los microprogramas radiofónicos, consistió en informar, sensibilizar e incentivar la reflexión en la comunidad universitaria y en el público en general, de una manera novedosa. ya que se utilizaba un medio de comunicación masiva.

En los 14 microprogramas realizados, se abordaron diversos temas en torno a la discusión sobre la calidad universitaria, sobre todo en lo referente a los procesos de autoevaluación, con fines de mejoramiento y lo acreditación. De esta manera, se trataron temas 
relacionados con el funcionamiento del CEA, fines y propósitos de la evaluación en la Universidad de Costa Rica, funcionamiento y características del Sistema Nacional de Acreditación de la Educación Superior (SINAES) y del Sistema Centroamericano de Evaluación y Acreditación de la Educación Superior (SICEVAES), cultura universitaria, conocimiento y gestión de los procesos de autoevaluación, acreditación y ejes transversales de la calidad como género, medio ambiente y discapacidad.

Para tener conocimiento acerca del impacto de esta actividad, en términos de sensibilización y difusión de los procesos de calidad y excelencia en la UCR, se requería conocer la opinión del público meta, respecto a la valoración de la pertinencia, tanto de la información, como de la forma en que el mensaje era presentado. Sin embargo, debido al corto tiempo en que se difundieron los programas, no se realizó la evaluación respectiva.

\subsubsection{Resultados de investigaciones}

A partir del año 2001, se inició una línea de investigación en el CEA con dos propósitos fundamentales: por un lado se buscó contribuir a sustentar la toma de decisiones de las autoridades universitarias, con una base científica y rigurosa, a saber: apoyar las decisiones de la Rectoría, la Vicerrectoría de Docencia y el Consejo Universitario. Por otra parte, los resultados de las investigaciones realizadas por el personal del DIEA, pretendían mejorar, sustantivamente, la calidad de las asesorías brindadas a las UA.

Las temáticas desarrolladas en las investigaciones, se pueden agrupar en tres áreas:

1. Área para sustentar la toma de decisiones institucionales. Dentro de esta área se encuentra el proyecto de Deserción universitaria, en donde se consideraron las cohortes de estudiantes del año 1993 a 1996; el proyecto de Indicadores de deserción universitaria, según áreas académicas del año 2000 al 2002 y el proyecto de los denominados Cursos no ponderables.

2. Área para el mejoramiento de la calidad de las asesorías brindadas por el personal del DIEA. En esta área se enmarcan los proyectos de investigación que se realizaron en el Curso de capacitación en métodos innovativos de autoevaluación del proyecto CAMINA/COSTA RICA 2002. Las investigaciones en las cuales participaron los siete representantes del CEA-DIEA, fueron las 
siguientes: estilo de desarrollo en Costa Rica y tendencias de acreditación; vinculación entre los procesos de autoevaluación- autorregulación y la acreditación con el proceso de planificación institucional; gestión de los procesos de autoevaluación; modelos de acreditación internacional: el caso de la CEAB y las carreras de Ingeniería en la Universidad de Costa Rica; evaluación del desempeño docente después de los procesos de autoevaluación y la acreditación.

Los resultados de estas investigaciones fueron incorporados en las capacitaciones del proyecto de calidad y excelencia, concretamente en los seminarios de capacitación del módulo de formación $\mathrm{y}$, además, fueron expuestos en las Jornadas de Investigación del año 2003, organizadas por la Vicerrectoría de Investigación.

3. Área para el mejoramiento de la docencia. En esta línea de investigación se ubica el proyecto sistema de evaluación docente. El proyecto denominado currículo inclusivo pretende incidir, directamente, en una perspectiva inclusiva desde la docencia, la investigación, el diseño curricular y, mediante la generación de indicadores de calidad de género, en la autoevaluación.

\subsection{Módulo de formación}

Ante la diversificación de las líneas de trabajo del DIEA, se sintió la necesidad de redefinir la función asesora que se brindaba a las UA, para que fueran éstas las protagonistas y responsables de la conducción de los procesos de autoevaluación. Dentro de esta reorientación, la persona asesora ofrecía un acompañamiento y algunas orientaciones técnicas que agilizaban la ejecución de los distintos momentos del proceso.

El módulo de formación se ideó con la intención de promover una nueva perspectiva de asesoramiento, dirigida a las personas que integraban las comisiones de autoevaluación de las UA, fueran estas docentes, estudiantes o personal administrativo, que recién habían iniciado el proceso de autoevaluación, o bien, las que se encontraban en etapas iniciales o intermedias.

Con estos módulos de formación, se visualizó la importancia de compartir con las UA, una perspectiva teórica, así como una propuesta metodológica de la organización integral del 
proceso de autoevaluación. Esto permitió que las personas pudieran visualizar la trayectoria total del proceso de autoevaluación, así como su complejidad, en términos de actores, interrelaciones, apoyos y limitaciones.

A los participantes en el curso, se les facilitaba la organización de sus procesos de autoevaluación, tomando en cuenta las especificidades propias de su UA. Este aprendizaje se facilitó mediante los conocimientos y experiencias compartidas, tanto por el personal asesor del DIEA, como por los docentes de las UA que ya habían realizado autoevaluaciones. Además se pretendía que, mediante los talleres y experiencias compartidas, las UA tuvieran herramientas y criterios básicos que les permitieran planear y desarrollar procesos de autoevaluación más independientes, en donde el papel asesor del DIEA fuera fundamentalmente de acompañamiento.

La siguiente figura muestra el esquema del módulo de formación:

Figura No. 5

PROYECTO PROMOCIÒN DE LA CALIDAD Y LA EXCELENCIA

EN LA UNIVERSIDAD DE COSTA RICA

Módulo de formación

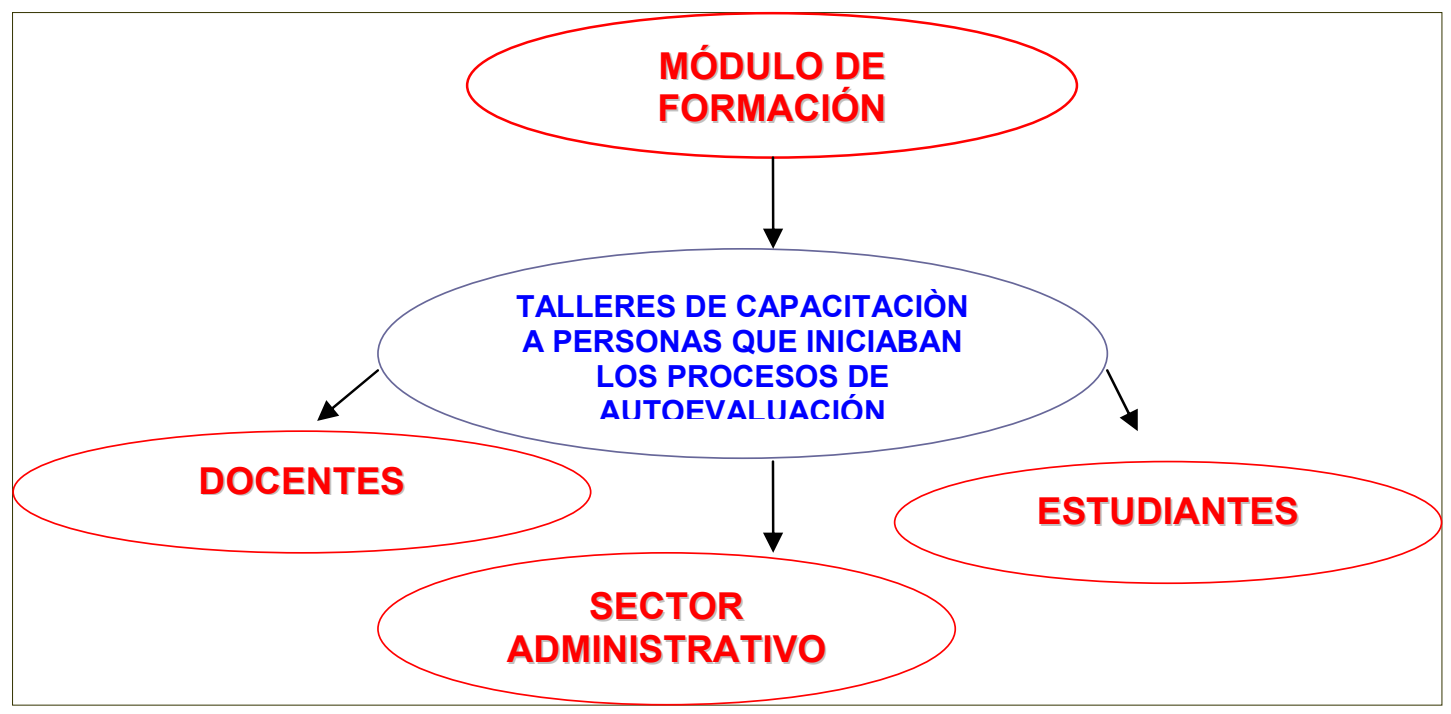

Elaboración propia. 


\subsubsection{Talleres de capacitación}

Mediante la organización de los temas tratados en los talleres de capacitación, se brindó una visión general de todo el proceso de autoevaluación y autorregulación, así como las acciones por seguir para la acreditación de los programas académicos. Dentro de este módulo de formación, se realizaron cuatro talleres y se capacitaron cerca de 200 personas de las diferentes sedes universitarias

El primer taller se organizó para las sedes universitarias y tuvo una duración de tres y medio días. Se realizó mediante las modalidades de charlas, taller y foro. En el taller se privilegió la discusión en torno a las situaciones concretas que enfrentaban las carreras en las sedes universitarias y sobre los problemas de autoevaluación. El tema del foro giró en torno a las agencias acreditadoras.

Las dos siguientes actividades se desarrollaron bajo la modalidad de seminarios, cada uno con una duración de dos días. Fueron diseñados para personas de distintas UA de la sede Rodrigo Facio, que habían tomado la decisión de iniciar un proceso de autoevaluación, así como aquellas que se encontraban en momentos iniciales o intermedios.

Todos los talleres se realizaron en la sede Rodrigo Facio y se estructuraron en cinco grandes ejes o temáticas, a saber:

a. Primer eje: Introducción general al proceso de evaluación:

En este eje, se presentaban los distintos abordajes teóricos de la evaluación y su desarrollo en el contexto internacional, así como la discusión o debate actual sobre el concepto de calidad en el ámbito académico. Finalmente, el CEA les presentaba a las personas participantes, la propuesta institucional sobre la autoevaluación y el tema de la calidad académica en la Universidad, a partir de los principios y propósitos contemplados en el Estatuto Orgánico y las políticas emitidas por el Consejo Universitario. Esta visión estaba permeada por la concepción humanista que caracteriza a la UCR.

b. Segundo eje: proceso de autoevaluación:

Con el propósito de mostrar la magnitud y la complejidad que implica la autoevaluación de una carrera, en este eje se proponían diferentes formas de organizar el trabajo interno, por medio de la división del proceso en etapas. También se brindaban orientaciones y 
sugerencias para la planificación y ejecución de la autoevaluación y de la vinculación de ésta con la planificación institucional. La finalidad era que estos procesos pudieran formar parte de la planificación general de la UCR y se pudiera contar con los recursos y apoyos necesarios para la conducción de la autoevaluación.

Las personas asesoras del DIEA proponían, en las charlas, que la autoevaluación fuera abordada como un proceso de investigación, en donde su objeto de estudio respondiera a las necesidades de mejoramiento y a los intereses académicos propios de cada UA. Para ello se recomendaba iniciar el proceso de autoevaluación con un prediagnóstico, realizado desde la UA, mediante el cual se determinaban las fortalezas, así como las áreas que debían mejorarse. Posteriormente, basado en esta información, la UA diseña su propuesta de autoevaluación. Esta propuesta debía planearse y ejecutarse, mediante las técnicas de recopilación y procesamiento de la información, propias de todo proceso de investigación académica.

c. Tercer eje: proceso de autorregulación:

Mediante este eje se buscaba brindar una orientación general para elaborar la planificación de la propuesta de autorregulación, de manera tal que tomaran en cuenta los resultados de la autoevaluación, las políticas universitarias y algunas técnicas de la planificación.

d. Cuarto eje: acreditación:

Conociendo las tendencias mundiales que favorecían la acreditación de carreras y programas, así como la aparición de agencias acreditadoras, tanto nacionales como internacionales, por medio de este eje, se rescataba la discusión universitaria en torno al tema, así como las ventajas y desventajas de la acreditación.

e. Quinto eje: experiencia de casos:

Este eje se diseñó partiendo del concepto de que una de las funciones de la persona asesora, es propiciar puntos de encuentro entre participantes con diferentes experiencias. Fue así cómo se favoreció el intercambio de experiencias vividas por personas que ya habían realizado los procesos de autoevaluación, recibido la visita de los evaluadores externos o bien que tuvieran experiencia en los procesos de autorregulación. 
Esta fue una de las actividades más enriquecedoras para los participantes, ya que les permitió a sus UA conocer, de una manera contextualizada y cercana, las diferentes actividades vividas por las personas que ya habían pasado por las etapas del proceso de autoevaluación.

\subsubsection{Evaluaciones de los talleres}

Con un instrumento desarrollado por el DIEA se realizó una evaluación de las diferentes actividades, talleres o seminarios. Los rubros que se evaluaron fueron los siguientes:

a. Nivel de utilidad: ¿en qué medida se consideran los temas tratados, útiles para las labores?

b. Nivel de satisfacción: ¿en qué medida se está satisfecho o satisfecha de haber participado en el taller?

c. Nivel de logro: este rubro se midió en cuatro dimensiones a saber, i. comprensión de conceptos, ii. exploración de nuevas ideas, iii. aclaración de confusiones y iv. ¿en qué medida la asistencia al taller, le permitió cambiar actitudes hacia la autoevaluación?

d. Cumplimiento de expectativas: ¿en qué medida el taller respondió a lo que las personas asistentes esperaban y a sus necesidades?

Los aspectos anteriores se debían valorar en una escala de 1 a 10 . Adicionalmente, se solicitó a las personas participantes que expresaran su opinión y que asignaran un adjetivo con el cual calificaran la actividad.

A manera de ejemplo, se cita el resumen de los resultados de la evaluación del taller realizado el 07 y 08 de octubre del año 2003, el cual fue elaborado por la señora Lorena Kikut, estadística del DIEA. Se obtuvieron los siguientes resultados de 23 cuestionarios aplicados:

1. El aspecto mejor evaluado es el nivel de utilidad del taller, al cual se le dieron puntajes que oscilaron entre 8 y 10 , para un promedio de 9.57 .

2. Los aspectos a los que se les dio una valoración más baja, fueron el cumplimiento de expectativas y la exploración de nuevas ideas, con promedios de 9.00 y 9.09 .

3. En promedio, a la actividad se le dio una calificación de 9.19. 
4. Las preguntas abiertas ofrecen una importante visión de las impresiones de los participantes hacia el taller. Algunas de las respuestas se refieren a:

- La necesidad de respetar el horario, tanto de inicio como el de cada intervención.

- La importancia de entregar material por escrito, lo que se traduciría en la disminución del tiempo de cada charla.

- Dotar a las exposiciones de más ejemplos y experiencias de unidades académicas que ya hayan pasado por el proceso de autoevaluación.

- Dar una mayor importancia al presupuesto del que disponen las unidades académicas para realizar los procesos de autoevaluación.

- Tomar en cuenta la poca posibilidad que se tiene de asistir dos días seguidos a una actividad de este tipo y el hecho de que las asambleas de escuela, por lo general, se realizan los días miércoles.

5. Los adjetivos dados por las personas participantes, valoran de manera muy positiva, la actividad, dentro de los que se tienen: excelente, bueno, muy bueno, útil, motivador, oportuno, positivo y pertinente.

En la siguiente tabla se agrupan los resultados de la evaluación del segundo taller realizado del 08 y 09 de abril del año 2003, en el Mini Auditorio de la Facultad de Letras. En esa ocasión se aplicaron 27 cuestionarios. 
Tabla No. 1

PROYECTO PROMOCIÒN DE LA CALIDAD Y LA EXCELENCIA EN LA UNIVERSIDAD DE COSTA RICA

Módulo de formación

Resultados de la evaluación del segundo taller

\begin{tabular}{|l|c|}
\hline \multicolumn{1}{|c|}{ ASPECTO } & $\begin{array}{c}\text { PROMEDIO } \\
\text { (escala 1 a 10) }\end{array}$ \\
\hline $\begin{array}{l}\text { Nivel de utilidad: ¿en qué medida considera útiles para } \\
\text { sus labores los temas tratados? }\end{array}$ & 9.4 \\
\hline $\begin{array}{l}\text { Nivel de satisfacción: ¿en qué medida está usted } \\
\text { satisfecho de haber participado en el taller? }\end{array}$ & 9.1 \\
\hline $\begin{array}{l}\text { Nivel de logro: ¿en qué medida considera usted que se } \\
\text { logró? }\end{array}$ & 8.9 \\
\hline a. Comprender conceptos & 9.1 \\
\hline b. Explorar nuevas ideas & 9.0 \\
\hline c. Aclarar confusiones & 9.0 \\
\hline d. Cambiar actitudes hacia la autoevaluación & 9.0 \\
\hline e. Cumplimiento de expectativas & \\
\hline
\end{tabular}

De los datos obtenidos, se deduce que la actividad fue considerada, como de una alta o muy alta utilidad para las labores que realizan las personas en sus respectivas Escuelas.

\section{Comentarios finales}

De la experiencia realizada dentro del Proyecto Promoción de la Calidad y la Excelencia en la Universidad de Costa Rica, se pueden extraer conclusiones importantes que retroalimentan el trabajo de asesoría del DIEA. De esta manera, dentro de los principales hallazgos encontrados como aprendizajes para las unidades académicas, se plantea que mediante el proyecto se favoreció:

- Estudiar, de manera más sistemática, los fundamentos teóricos del proceso de autoevaluación y tener un conocimiento conceptual más detallado de los temas de la autoevaluación y acreditación.

- Reafirmar la visión de la autoevaluación, como un proceso de reflexión autocrítico y de valoración del quehacer diario. 
- Visualizar a la autorregulación, como un proceso posterior a la autoevaluación que permite organizar, de manera sistemática, el funcionamiento de la unidad académica.

- Valorar las experiencias acumuladas por parte de las diferentes unidades académicas que han realizado el proceso de autoevaluación y las que ya tienen carreras acreditadas.

- Conocer y valorar ideas y propuestas, acerca de algunos mecanismos de gestión que contribuyen a agilizar los procesos de autoevaluación.

- Apropiarse de los conocimientos teóricos y metodológicos que permiten realizar procesos de autoevaluación de manera más autónoma.

- Favorecer el aprendizaje entre unidades académicas.

- Establecer vínculos de cooperación entre unidades académicas que permiten intercambiar información y metodologías utilizadas, durante los procesos de autoevaluación.

- Brindar un espacio para plantear preguntas e inquietudes a personas de otras unidades académicas y no solo al personal asesor del DIEA.

- Vincular los procesos de autoevaluación y mejoramiento con la planificación institucional, por medio de la formulación de proyectos en las áreas de investigación, innovación docente, acción social, vida estudiantil y administración.

Por otra parte, el proyecto permitió a los y las funcionarias del DIEA:

- Sistematizar sus conocimientos, experiencias y experticias profesionales.

- Proyectar, a la comunidad universitaria, un enfoque de asesoramiento orientado a la promoción de las relaciones horizontales.

- Favorecer las relaciones de cooperación entre las unidades académicas.

- Recoger una serie de recomendaciones que pueden agilizar la función asesora en los temas de la autoevaluación.

- Facilitar el trabajo de asesoría, puesto que las UA ya tienen conocimientos previos cuando inician el proceso de autoevaluación.

- Proyectarse profesionalmente en el ámbito universitario y perfilarse como la instancia intermediaria que contribuye a la discusión que se desarrolla en las UA, en torno a los temas de autoevaluación y acreditación. 
- Incentivar la discusión interna acerca de las implicaciones de los procesos de autoevaluación y la acreditación de carreras en los diferentes ámbitos del quehacer institucional.

De manera adicional, el desarrollo del proyecto favoreció también una mayor unión y compañerismo entre los y las funcionarias del DIEA, ya que se favoreció el trabajo en equipo, mediante la elaboración conjunta de charlas, conferencias y la preparación de materiales que son utilizados como material en las asesorías que se brindan.

Finalmente, se debe resaltar la importancia de que los procesos de capacitación en el ámbito académico sean planificados y respondan a las demandas y necesidades de la comunidad universitaria. De igual manera, se debe considerar la posibilidad de que iniciativas como la sistematizada en este artículo, que demuestran tener resultados enriquecedores, tanto para el público destinatario, como para los ejecutores, tengan continuidad dentro de los procesos de planificación de las instancias universitarias. 


\section{REFERENCIAS}

Bolívar, A. (1999). El asesoramiento curricular a los establecimientos educacionales: de los enfoques técnicos a la innovación y desarrollo interno. Recuperado el 18 de abril de 2005, de http://csociales.uchile.cl/publicaciones/enfoques/03/edu01.htm

Centro de Evaluación Académica. (s.f.) Documentos contenidos en el archivo del Proyecto Promoción de la Calidad y la Excelencia en la Universidad de Costa Rica. 2002-2003

Chavarría, E. (2001). Papel del asesor CEA- DEDUN. Conferencia.

Erazo, I. (2005). La evaluación y la metaevaluación: dos procesos de la estrategia de gestión de la calidad de la Educación Superior centroamericana. Simposio internacional: de la evaluación y acreditación al mejoramiento de la calidad de la Educación Superior (Del 09 al 11 de marzo, 2005). Costa Rica: CSUCA-INWENTRIACES-CCA-UNED.

Hernández, F. (1999). La construcción del saber del asesor. Revista de Teoría, Investigación y Práctica Educativa Qurrículum. Q-No.5. Recuperado el 18 de abril de 2005 de http://www.quadernsdigitals.net/index.php?accionMenu=hemeroteca.VisualizaArticulol $\underline{\text { U.visualiza\&articulo } \mathrm{id}=2660}$

Segovia, J. (2003). El asesoramiento a centros educativos, una cuestión de saber, poder e identidad. Recuperado el 22 de abril de 2005 de http://www.ugr.es/refpro/Rev71COL1.pdf

Vargas, A. (2001). La cultura evaluativa en la Universidad de Costa Rica. Su construcción desde la evaluación docente. Tesis para optar por el grado de doctora en Educación. Universidad de Costa Rica. Costa Rica.

Wesseler, M. (2004). La confianza importa: cómo la cultura institucional puede instigar la gestión de la calidad y del cambio en la Educación Superior. Programa internacional de gestión de la calidad y del cambio en la Educación Superior UniCambio XXI (pp. 85-94). Módulo 2. Eschborn, Alemania: GTZ. 